\title{
The Design of GP 2
}

\author{
Detlef Plump \\ Department of Computer Science \\ The University of York, UK
}

\begin{abstract}
This papers defines the syntax and semantics of GP 2, a revised version of the graph programming language GP. New concepts are illustrated and explained with example programs. Changes to the first version of GP include an improved type system for labels, a built-in marking mechanism for nodes and edges, a more powerful edge predicate for conditional rule schemata, and functions returning the indegree and outdegree of matched nodes. Moreover, the semantics of the branching and loop statement have been simplified to allow their efficient implementation.
\end{abstract}

\section{Introduction}

GP is an experimental nondeterministic programming language for high-level problem solving in the domain of graphs. The language is based on conditional rule schemata for graph transformation and has a simple syntax and semantics, to facilitate both understanding by programmers and formal reasoning on programs. The original version of GP (also referred to as GP 1 from now on) is defined in [7, 8] and its protoype implementation is described in [4].

Motivated by case studies in GP programming, the following changes and extensions feature in GP 2:

- There are new types atom and list, the former representing the union of integers and character strings, the latter lists of atoms. Variables of these types can be declared in rule schemata.

- Rule schemata can mark nodes and edges graphically.

- Conditional rule schemata can check, by means of the edge predicate, whether there exists an edge with a particular label between two matched nodes.

- The indegree or outdegree of a matched node can be accessed and used in the labels or in the condition of a rule schema.

- The if-then-else statement of GP 1 is complemented by a try-then-else command whose then-part is executed on the graph resulting from the try-part. Also, a new or command provides explicit nondeterministic choice between subprograms.

- Failure in evaluating the condition of a branching statement or the body of a loop does no longer enforce backtracking, in order to allow an efficient implementation of branching and looping.

The rest of this paper is organised as follows. In Section 2, the graph transformation approach underlying GP is briefly reviewed, viz. the double-pushout approach with relabelling. Section 3 introduces conditional rule schemata, the building blocks of GP programs. The semantics of conditional rule schemata is defined in Section 4 In Section 5 new features of GP 2 are demonstrated and explained by example programs. A formal operational semantics for GP 2 is presented and discussed in Section 6 Section 7 concludes by summarising GP's revision and addressing topics for future work. The Appendix lists the inference rules of the semantics of Section 6 


\section{Graphs and Graph Transformation}

Graph transformation in GP is based on the double-pushout approach with relabelling [3]. This framework deals with partially labelled graphs whose definition is recalled below. In this section, we treat the label alphabet as a parameter because in subsequent sections we need different alphabets: graphs in rule schemata are labelled with expressions while graphs on which GP programs operate (also referred to as host graphs) are labelled with lists composed of integers and strings.

A graph over a label alphabet $\mathscr{C}$ is a system $G=\left(V_{G}, E_{G}, s_{G}, t_{G}, l_{G}, m_{G}\right)$, where $V_{G}$ and $E_{G}$ are finite sets of nodes (or vertices) and edges, $s_{G}, t_{G}: E_{G} \rightarrow V_{G}$ are the source and target functions for edges, $l_{G}: V_{G} \rightarrow \mathscr{C}$ is the partial node labelling function and $m_{G}: E_{G} \rightarrow \mathscr{C}$ is the (total) edge labelling function. Given a node $v$, we write $l_{G}(v)=\perp$ to express that $l_{G}(v)$ is undefined. Graph $G$ is totally labelled if $l_{G}$ is a total function. We write $\mathscr{G}\left(\mathscr{C}_{\perp}\right)$ and $\mathscr{G}(\mathscr{C})$ for the class of graphs resp. totally labelled graphs over $\mathscr{C}$.

Unlabelled nodes will occur only in the interfaces of rules and are necessary in the double-pushout approach to relabel nodes. There is no need to relabel edges as they can always be deleted and reinserted with different labels.

A graph morphism $g: G \rightarrow H$ between graphs $G, H$ in $\mathscr{G}\left(\mathscr{C}_{\perp}\right)$ consists of two functions $g_{V}: V_{G} \rightarrow V_{H}$ and $g_{E}: E_{G} \rightarrow E_{H}$ that preserve sources, targets and labels; that is, $s_{H} \circ g_{E}=g_{V} \circ s_{G}, t_{H} \circ g_{E}=g_{V} \circ t_{G}$, $m_{H} \circ g_{E}=m_{G}$, and $l_{H}(g(v))=l_{G}(v)$ for all $v$ such that $l_{G}(v) \neq \perp$. Morphism $g$ is an inclusion if $g(x)=x$ for all nodes and edges $x$. It is injective (surjective) if $g_{V}$ and $g_{E}$ are injective (surjective). It is an isomorphism if it is injective, surjective and satisfies $l_{H}\left(g_{V}(v)\right)=\perp$ for all nodes $v$ with $l_{G}(v)=\perp$. In this case $G$ and $H$ are isomorphic, which is denoted by $G \cong H$.

A rule $r=\langle L \leftarrow K \rightarrow R\rangle$ consists of two inclusions $K \rightarrow L$ and $K \rightarrow R$ such that $L, R$ are graphs in $\mathscr{G}(\mathscr{C})$ and $K$, the interface of $r$, is a graph in $\mathscr{G}\left(\mathscr{C}_{\perp}\right)$. Intuitively, an application of $r$ to a graph will remove the items in $L-K$, preserve $K$, add the items in $R-K$, and relabel the unlabelled nodes in $K$.

Definition 1 (Rule application). Let $r=\langle L \leftarrow K \rightarrow R\rangle$ be a rule, $G$ a graph in $\mathscr{G}(\mathscr{C})$, and $g: L \rightarrow G$ an injective graph morphism satisfying the dangling condition: no node in $g(L)-g(K)$ is incident to an edge in $G-g(L)$. We write $G \Rightarrow_{r, g} H$ if $H$ is isomorphic to the graph that is constructed from $G$ as follows:

1. Remove all nodes and edges in $g(L)-g(K)$, obtaining a graph $D$.

2. Add disjointly to $D$ all nodes and edges from $R-K$, keeping their labels. For $e \in E_{R}-E_{K}, s_{H}(e)$ is $s_{R}(e)$ if $s_{R}(e) \in V_{R}-V_{K}$, otherwise $g_{V}\left(s_{R}(e)\right)$. Targets are defined analogously.

3. For each unlabelled node $v$ in $K, l_{H}\left(g_{V}(v)\right)$ becomes $l_{R}(v)$.

Figure 1 shows an example of a rule application. The rule in the upper row is applied to the left graph of the lower row, resulting in the right graph of the lower row. (For simplicity, we assume that all edge labels are the same and hence omit them.) The node identifiers 1 and 2 in the rule specify the inclusions of the interface. The middle graph of the lower row is obtained from graph $D$ of Definition 1 by making all nodes unlabelled that are images of unlabelled nodes in $K$. Then the diagram represents a double-pushout in the category of graphs over $\mathscr{C}_{\perp}$ (see [3]).

\section{Syntax of Rule Schemata}

Conditional rule schemata are the principal programming construct in GP. Figure 2 shows an (artificial) example for the declaration of a rule schema containing some of the new features of GP 2. 


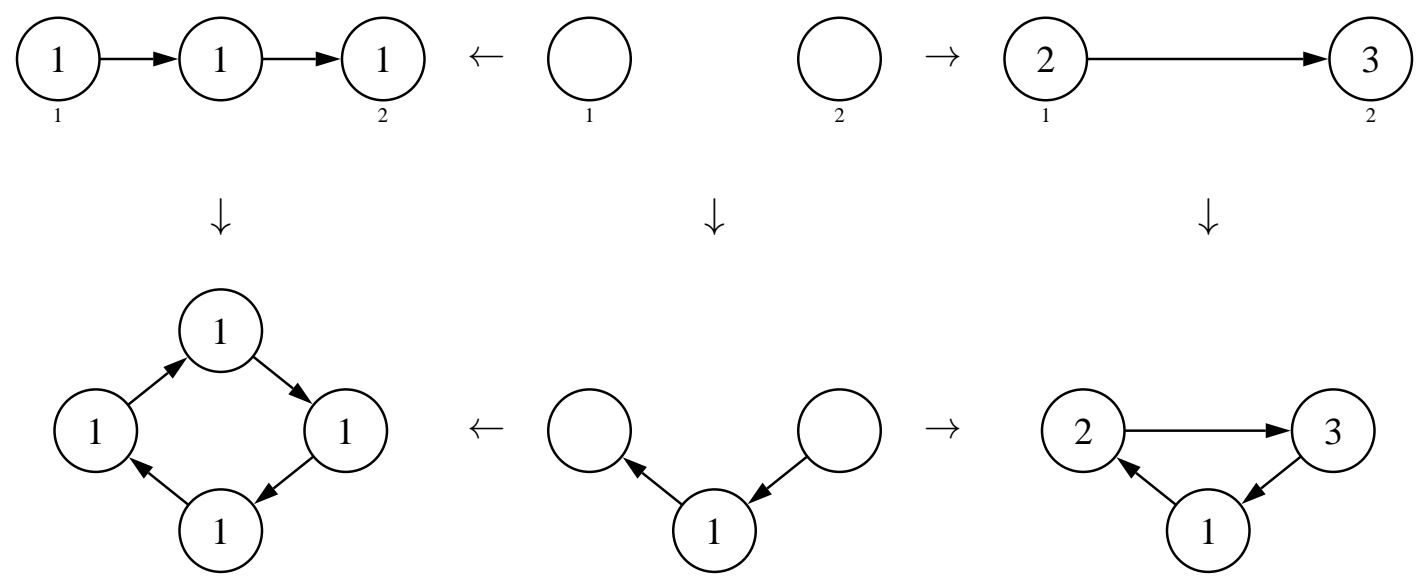

Figure 1: A rule application

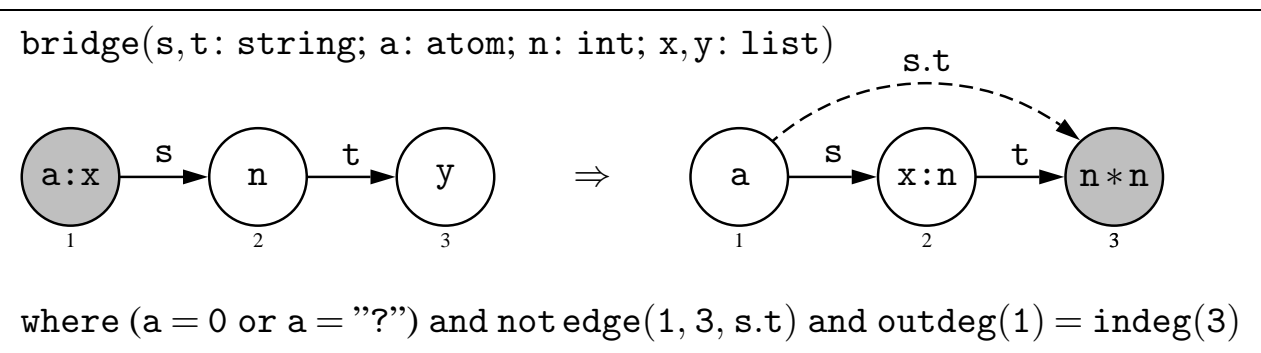

Figure 2: Declaration of a conditional rule schema

Besides the types int and string of GP 1 , there are the new types atom and list. Type atom is the union of int and string, and list is the type of a (possibly empty) list of atoms. Given lists $\mathrm{x}$ and $\mathrm{y}$, we write $\mathrm{x}: \mathrm{y}$ for the concatenation of $\mathrm{x}$ and $\mathrm{y}$. The colon replaces the underscore '_, of GP 1 for better readability. Also, the empty list empty is now allowed (not to be confused with the empty character string "'). When drawing graphs, we represent the empty list by omitting the word empty. (Confusion with unlabelled nodes is not possible as long as we consider graphs on the left or right of a rule schema, or host graphs. This is because these graphs are totally labelled.)

We identify lists of length one with their contents and hence get the syntactic and semantic subtype relationships shown in Figure 3. This is why we can form list expressions such as a: $\mathrm{x}$ and $\mathrm{x}: \mathrm{n}$ in Figure 2. where $\mathrm{x}$ is a list, $\mathrm{a}$ an atom and $\mathrm{n}$ an integer. For the same reason, equations in the condition such as $\mathrm{a}=0$ or $\mathrm{a}=$ "?" can compare expressions of arbitrary list subtypes.

Expressions in the left-hand side of a rule schema need no longer be constants or variables. Composite expressions such as a: $\mathrm{x}$ in Figure 2 are allowed if there is no ambiguity in matching individual variables with values in host graph labels. Similarly, the new dot operator '.' for string concatenation can be used in left-hand labels. (The exact condition for left-hand expressions is given in Definition 2)

The new functions indeg and outdeg access the indegree resp. outdegree of a left-hand node in the host graph. These operators may occur in the labels and the condition of a rule schema. Moreover, the binary edge predicate of GP 1 has now an optional third argument specifying the label of a possible edge 

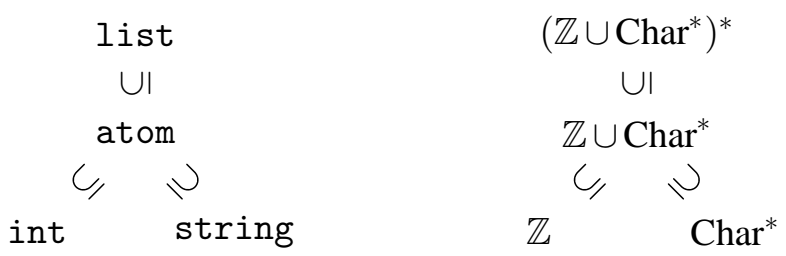

Figure 3: Subtype hierarchy for lists

between the given nodes. For example, the subcondition not edge $(1,3$, s.t) in Figure 2 demands that there must not be an edge from node 1 to node 3 with label s.t (where the strings denoted by $s$ and $t$ are determined by matching the left-hand graph).

Finally, GP 2 allows to mark nodes and edges graphically. For example, the outermost nodes in Figure 2 are marked by a grey shading, and the dashed arrow between nodes 1 and 3 in the right graph represents a marked edge. Marking is formalised below by defining labels as pairs of lists and boolean values, where a boolean value indicates whether a node or edge is marked or not.

Figure 4 and Figure 5 give grammars in Extended Backus-Naur Form defining the abstract syntax of the labels and the condition of a rule schema. These grammars are ambiguous; in examples we use parentheses to disambiguate expressions if necessary. In the next section, the abstract syntax is used in defining the semantics of rule schemata.

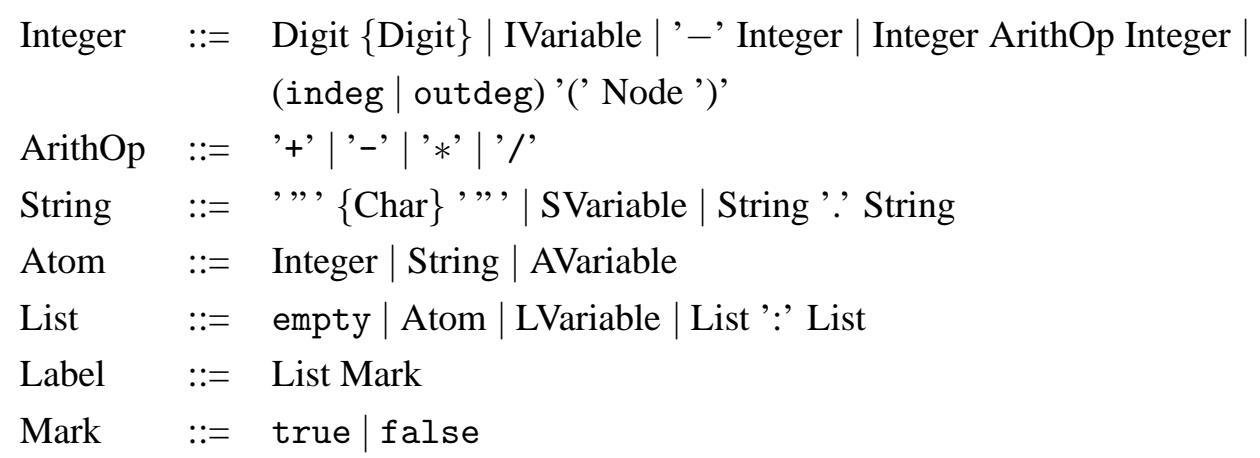

Figure 4: Abstract syntax of rule schema labels

The grammar in Figure 4defines four syntactic categories of expressions which can occur in a rule schema: Integer, String, Atom and List, where Integer and String are subsets of Atom which in turn is a subset of List. We assume that Node is the set of node identifiers occurring in the rule schema, which must be the same for the left and the right graph $(\{1,2,3\}$ in Figure 2). Moreover, IVariable, SVariable, AVariable and LVariable are the sets of variables of type int, string, atom and list that occur in the rule schema. These categories are disjoint since each variable must be declared with a unique type (see Figure 2). The mark components of labels are represented graphically rather than textually.

The values of variables at execution time are determined by graph matching, hence we require that expressions in the left graph of a rule schema must have a simple shape. 
Definition 2 (Simple list). An expression $e \in$ List is simple if

(1) $e$ contains no arithmetic operators,

(2) $e$ contains at most one occurrence of a list variable, and

(3) each occurrence of a string expression in $e$ contains at most one occurrence of a string variable.

For example, given the variable declarations of Figure 2, a: $x$ and "no". $\mathrm{s}: \mathrm{y}: \mathrm{t}$ are simple expressions whereas $\mathrm{x}: \mathrm{y}$ and $\mathrm{s} . \mathrm{t}$ are not simple.

The syntax of a rule schema condition is defined by the grammar in Figure 5. New features are the predicates int, string and atom, which allow to check whether an expression belongs to a subtype of list, and equations between arbitrary list expressions. Also, the edge predicate can have a third parameter specifying the list component of an edge label.

$$
\begin{aligned}
\text { Condition }::= & \text { Type '('List ')' | List ('=' | ' ! =') List | } \\
& \text { Integer RelOp Integer } \mid \\
& \text { edge '(' Node ',' Node [', List] ')' | } \\
& \text { not Condition | Condition (and | or) Condition } \\
\text { Type } \quad::= & \text { int } \mid \text { string } \mid \text { atom } \\
\text { RelOp } \quad::= & \text { '> |'>='|'<'|'<=' }
\end{aligned}
$$

Figure 5: Abstract syntax of rule-schema condition

Definition 3 (Conditional rule schema). A rule schema $\langle L \leftarrow K \rightarrow R\rangle$ consists of two inclusions $K \rightarrow L$ and $K \rightarrow R$ such that $L, R$ are graphs in $\mathscr{G}$ (Label) and $K$ consists of unlabelled nodes only. We require that all list expressions in $L$ are simple and that all variables occurring in $R$ also occur in $L$. A conditional rule schema $\langle L \leftarrow K \rightarrow R, c\rangle$ consists of a rule schema $\langle L \leftarrow K \rightarrow R\rangle$ and a condition $c \in$ Condition such that all variables occurring in $c$ also occur in $L$.

When a conditional rule schema is declared, as in Figure 2 graph $K$ is implicitly represented by the node identifiers in $L$ and $R$ (which much coincide). Hence nodes without identifiers in $L$ are to be deleted and nodes without identifiers in $R$ are to be created.

The requirement that all variables in $R$ must also occur in $L$ ensures that for a given match of $L$ in a host graph, applying $r$ produces a unique graph (up to isomorphism). Similarly, the evaluation of $c$ has a unique result if all its variables occur in $L$.

\section{Semantics of Rule Schemata}

While the left and right graph of a rule schema are labelled with elements from the syntactic category Label, host graphs are labelled with values from the following semantic domain $\mathscr{L}$ :

$$
\mathscr{L}=\left(\mathbb{Z} \cup \text { Char }^{*}\right)^{*} \times \mathbb{B}
$$

where $\mathbb{B}=\{$ true, false $\}$. Hence semantic labels are sequences consisting of integers and character strings 1 , paired with boolean values. As in the case of syntactic labels, the individual elements of a

\footnotetext{
${ }^{1}$ We assume that Char is a fixed set of characters.
} 
sequence are separated by colons, the empty sequence is represented by "white space", and the boolean value true is represented graphically by shading resp. dashed lines.

The application of a rule schema $r$ with condition $c$ to a graph $G$ in $\mathscr{G}(\mathscr{L})$ proceeds roughly as follows:

1. Match the left graph $L$ of $r$ with a subgraph of $G$, ignoring labels, by means of a premorphism $g: L \rightarrow G$.

2. Check whether there is an assignment $\alpha$ of values to variables such that after evaluating the expressions in $L, g$ is label-preserving.

3. Check whether the condition $c$ evaluates to true.

4. Apply the rule $r^{g, \alpha}$, obtained from $r$ by evaluating all expressions in the left and right graph, to $G$.

For example, Figure 6 shows an application of the rule schema bridge of Figure 2 The upper half of the diagram represents the instantiation of bridge according to premorphism $g$ and the following assignment $\alpha$ : $\mathrm{a} \mapsto 0, \mathrm{x} \mapsto 1: 2, \mathrm{n} \mapsto 3, \mathrm{y} \mapsto 4, \mathrm{~s} \mapsto$ "o", $\mathrm{t} \mapsto " k$ ". The lower half of the diagram represents the application of the instance bridge ${ }^{g, \alpha}$ according to $g$. Note that the application condition of bridge (see Figure 2) is satisfied with respect to $g$ and $\alpha$.
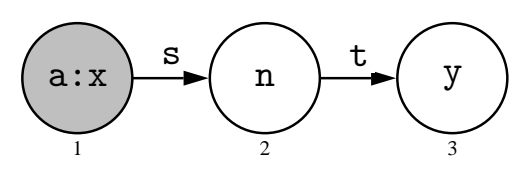

$\downarrow \alpha, g$

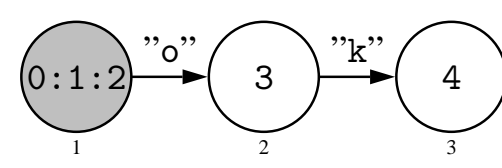

$\downarrow g$

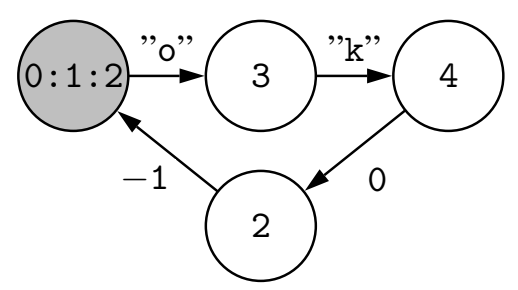

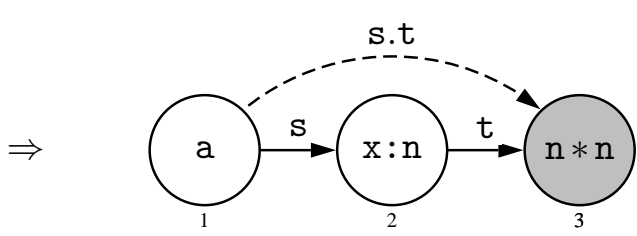

$\downarrow \alpha, g$

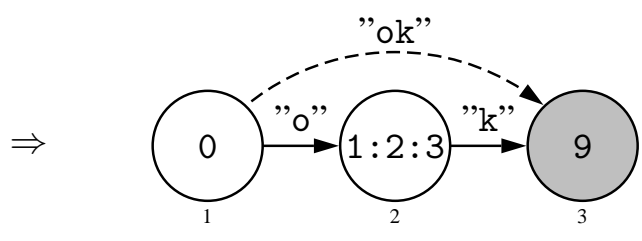

$\downarrow$

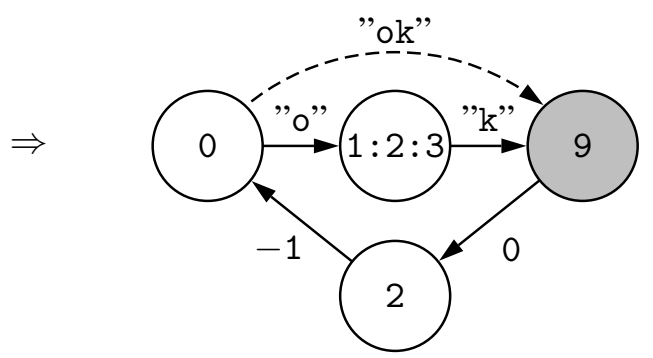

Figure 6: An application of the rule schema bridge of Figure 2

In the remainder of this section, we make the above four steps precise. Consider a conditional rule schema $r=\langle L \leftarrow K \rightarrow R, c\rangle$. Given graphs $G$ in $\mathscr{G}($ Label $)$ and $H$ in $\mathscr{G}(\mathscr{L})$, a premorphism $g: G \rightarrow H$ 
consists of two functions $g_{V}: V_{G} \rightarrow V_{H}$ and $g_{E}: E_{G} \rightarrow E_{H}$ that preserve sources and targets: $s_{H} \circ g_{E}=$ $g_{V} \circ s_{G}$ and $t_{H} \circ g_{E}=g_{V} \circ t_{G}$.

An assignment is a family of mappings $\alpha=\left(\alpha_{X}\right)_{X \in\{\mathrm{I}, \mathrm{S}, \mathrm{A}, \mathrm{L}\}}$ where $\alpha_{\mathrm{I}}$ : IVariable $\rightarrow \mathbb{Z}, \alpha_{\mathrm{S}}:$ SVariable $\rightarrow$ Char* $\alpha_{\mathrm{A}}$ : AVariable $\rightarrow \mathbb{Z} \cup$ Char $^{*}$ and $\alpha_{\mathrm{L}}$ : LVariable $\rightarrow \mathscr{L}$. We sometimes omit the subscripts of these mappings as exactly one of them is applicable to a given variable.

Given a premorphism $g: L \rightarrow G$, an assignment $\alpha$ and a label $l=e m$ with $e \in$ List and $m \in$ \{true, false , the value $l^{g, \alpha} \in \mathscr{L}$ is the pair $\left\langle e^{g, \alpha}\right.$, true $\rangle$ if $m=$ true and $\left\langle e^{g, \alpha}\right.$, false $\rangle$ otherwise.

The value $e^{g, \alpha} \in\left(\mathbb{Z} \cup \mathrm{Char}^{*}\right)^{*}$ is inductively defined. If $e=$ empty, then $e^{g, \alpha}$ is the empty sequence. If $e$ has the form $d_{1} \ldots d_{n}(n \geq 1)$ with digits $d_{1}, \ldots, d_{n}$, or has the form " $c_{1} \ldots c_{n}$ " $(n \geq 0)$ with characters $c_{1}, \ldots, c_{n}$, then $e^{g, \alpha}$ is the unique integer in $\mathbb{Z}$ resp. character string in Char* represented by $e$. (Note that the empty character string and empty ${ }^{g, \alpha}$ are different values.) If $e$ is a variable, then $e^{g, \alpha}=\alpha(e)$.

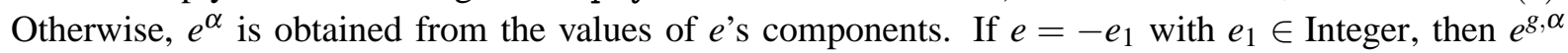
is the integer opposite to $e_{1}^{g, \alpha}$. If $e$ has the form $e_{1} \oplus e_{2}$ with $\oplus \in$ ArithOp and $e_{1}, e_{2} \in$ Integer, then $e^{g, \alpha}=e_{1}^{g, \alpha} \oplus_{\mathbb{Z}} e_{2}^{g, \alpha}$ where $\oplus_{\mathbb{Z}}$ is the integer operation represented by $\oplus_{2}$ If $e$ has the form indeg $(n)$ or outdeg $(n)$, with $n \in$ Node, then $e^{g, \alpha}$ is the indegree resp. outdegree of the node $g_{V}(n)$ in $G$. Finally, if $e=e_{1} . e_{2}$ with $e_{1}, e_{2} \in$ String or $e=e_{1}: e_{2}$ with $e_{1}, e_{2} \in$ List, then $e^{g, \alpha}$ is the concatenation of $e_{1}^{g, \alpha}$ and $e_{2}^{g, \alpha}$.

The value $c^{g, \alpha} \in \mathbb{B}$ of the condition $c$ is also inductively defined. If $c$ has the form $\operatorname{int}\left(e_{1}\right)$ with $e_{1} \in$ List, then $c^{g, \alpha}=$ true if and only if $e_{1}^{g, \alpha} \in \mathbb{Z}$. Similarly, if $c$ has the form $\operatorname{string}\left(e_{1}\right)$ or atom $\left(e_{1}\right)$, then $c^{g, \alpha}=$ true if and only if $e_{1}^{g, \alpha} \in$ Char* $^{*}$ resp. $e_{1}^{g, \alpha} \in \mathbb{Z} \cup$ Char*. If $c$ has the form $e_{1}=e_{2}$ or $e_{1} !=e_{2}$ with $e_{1}, e_{2} \in$ List, then $c^{g, \alpha}=$ true if and only if $e_{1}^{g, \alpha}=e_{2}^{g, \alpha}$ resp. $e_{1}^{g, \alpha} \neq e_{2}^{g, \alpha}$. If $c$ has the form $e_{1} \bowtie e_{2}$ with $\bowtie \in$ RelOp and $e_{1}, e_{2}$ in Integer, then $c^{g, \alpha}=$ true if and only if $e_{1}^{g, \alpha} \bowtie_{\mathbb{Z}} e_{2}^{g, \alpha}$ where $\bowtie_{\mathbb{Z}}$ is the integer relation represented by $\bowtie$.

If $c$ has the form edge $(m, n)$ with $m, n \in$ Node, then $c^{g, \alpha}=$ true if and only if there is an edge in $G$ from $g_{V}(m)$ to $g_{V}(n)$. Similarly, if $c$ has the form edge $(m, n, e)$ with $m, n \in$ Node and $e \in$ List , then $c^{g, \alpha}=$ true if and only if there is an edge from $g_{V}(m)$ to $g_{V}(n)$ with a label whose list component is $e^{g, \alpha}$.

If $c$ has the form not $c_{1}$ with $c_{1} \in$ Condition, then $c^{g, \alpha}=$ true if and only if $c_{1}^{g, \alpha}=$ false. Finally, if $c$ has the form $c_{1}$ and $c_{2}$ with $c_{1}, c_{2} \in$ Condition, then $c^{g, \alpha}=$ true if and only if $c_{1}^{g, \alpha}=$ true $=c_{2}^{g, \alpha}$, and if $c$ has the form $c_{1}$ or $c_{2}$, then $c^{g, \alpha}=$ true if and only $c_{1}^{g, \alpha}=$ true or $c_{2}^{g, \alpha}=$ true.

We call $r^{g, \alpha}=\left\langle L^{g, \alpha} \leftarrow K \rightarrow R^{g, \alpha}\right\rangle$ the instance of $r$ with respect to $g$ and $\alpha$, where $L^{g, \alpha}$ and $R^{g, \alpha}$ are obtained from $L$ and $R$ by replacing each label $l$ with $l^{g, \alpha}$. Note that $r^{g, \alpha}$ is a graph transformation rule over $\mathscr{L}$, in the sense of Section 2, We can now define the application of conditional rule schemata to graphs in $\mathscr{G}(\mathscr{L})$.

Definition 4 (Rule-schema application). Given a conditional rule schema $r=\langle L \leftarrow K \rightarrow R, c\rangle$ and graphs $G, H$ in $\mathscr{G}(\mathscr{L})$, we write $G \Rightarrow_{r, g} H$ (or just $G \Rightarrow_{r} H$ ) if there are a premorphism $g: L \rightarrow G$ and an assignment $\alpha$ such that

(1) $g$ is a graph morphism $L^{g, \alpha} \rightarrow G$,

(2) $c^{g, \alpha}=$ true, and

(3) $G \Rightarrow_{r, \alpha, g} H$.

Here $G \Rightarrow r_{r^{g, \alpha}, g} H$ denotes the application of $r^{g, \alpha}$ with match $g$ to $G$, as defined in Section 2 Note that we use $\Rightarrow$ for the application of both rule schemata and rules, to avoid an inflation of symbols. Given a set $\mathscr{R}$ of conditional rule schemata, we write $G \Rightarrow_{\mathscr{R}} H$ if $G \Rightarrow_{r} H$ for some conditional rule schema $r$ in $\mathscr{R}$.

\footnotetext{
${ }^{2}$ The effect of dividing by zero is undefined, that is, left to the implementation.
} 
The following proposition shows that given a rule schema $r$, a premorphism from the left-hand graph of $r$ to $G$ induces at most one instance of $r$ that can be applied with match $g$.

Proposition. Given a conditional rule schema $r=\langle L \leftarrow K \rightarrow R, c\rangle$ and a premorphism $g: L \rightarrow G$, there exists at most one assignment $\alpha$ such that $g$ is a graph morphism $L^{g, \alpha} \rightarrow G$.

The proof of this property relies on the fact that the left-hand graph $L$ contains only simple expressions.

\section{Programs}

The syntax of graph programs is the same as in GP 1, except for the syntax of rule schemata and the new constructs try_then_else and or. Figure 7 shows the abstract syntax of GP 2 programs. As before, a program consists of a number of declarations of conditional rule schemata and macros, and exactly one declaration of a main command sequence. The identifiers of category RuleId occurring in a RuleSetCall refer to declarations of conditional rule schemata in category RuleDecl (see previous sections).

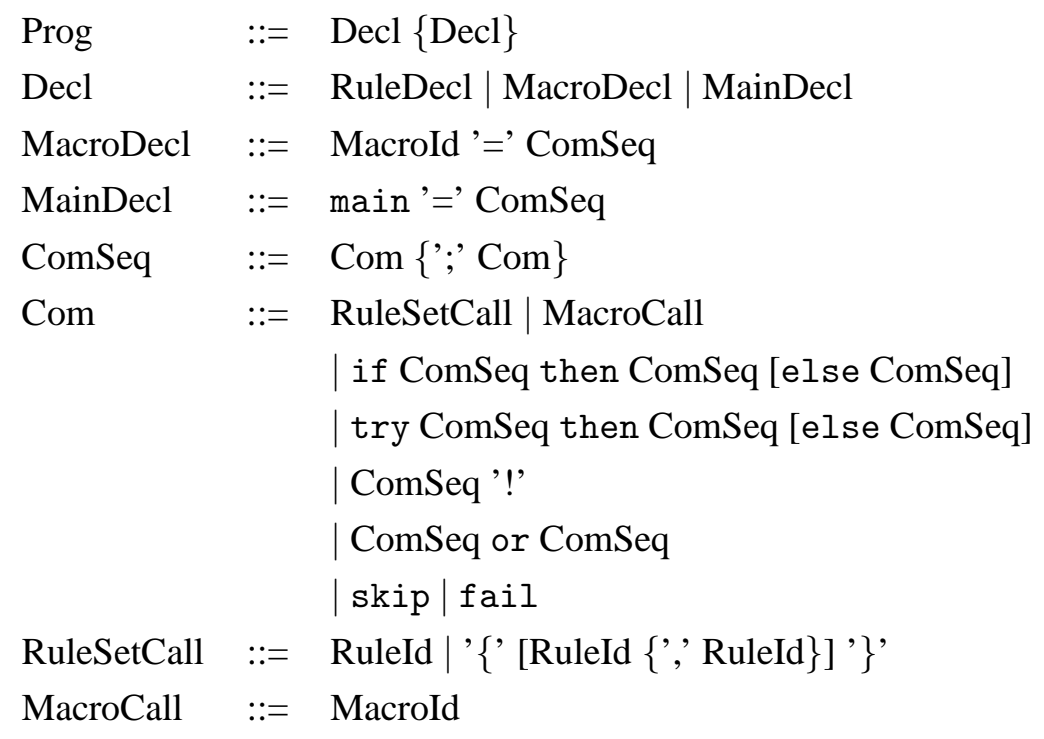

Figure 7: Abstract syntax of programs

In the next section it is shown that the commands or, skip and fail can be expressed through the other commands. Hence the core of GP includes only the call of a set of conditional rule schemata (RuleSetCall), sequential composition (';'), the if-then-else statement, the try-then-else statement and as-long-as-possible iteration ('!'). Before formally defining the semantics of programs, we discuss some example programs to illustrate the use of the new features of GP 2.

Example 1 (Checking connectedness). A graph is connected if there is an undirected path between each two nodes, that is, a sequence of consecutive edges whose directions don't matter. The program in Figure 8 checks whether an arbitrary input graph $G$ is connected and, depending on the result, executes either program $P$ or program $Q$ on $G$.

Connectedness is checked by picking some node, marking it, and propagating node marks along edges as long as possible. Then an application of the rule schema unmarked tests whether any unmarked 
nodes are left. If this is the case, then the macro disconnected succeeds and program $Q$ is executed, otherwise disconnected fails and program $P$ is executed.

It is important to note that $P$ or $Q$ is executed on the input graph whereas the graph resulting from the test is discarded. The precise semantics of the branching command is given in Section 6

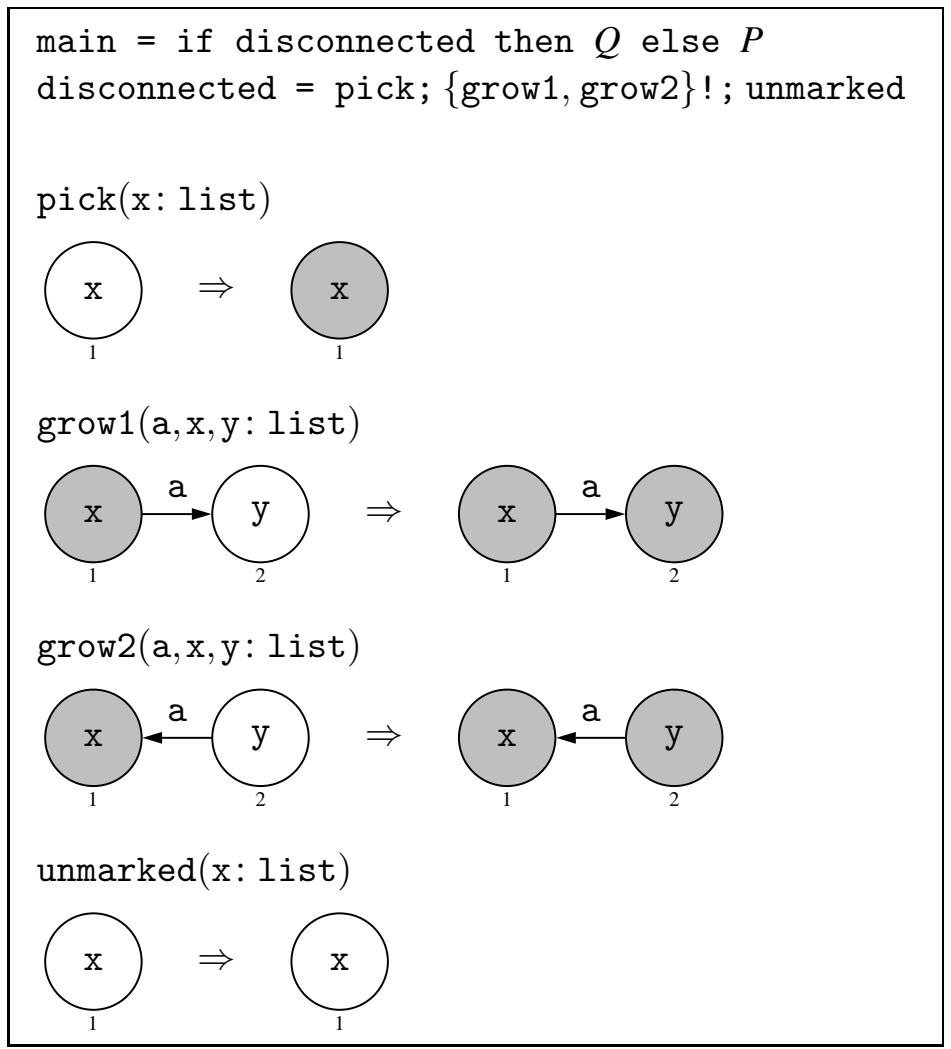

Figure 8: A program for checking connectedness

Example 2 (Recognising acyclic graphs). A graph is acyclic if it does not contain a directed cycle. The program in Figure 9 checks whether an unmarked input graph $G$ is acyclic and, depending on the result, executes either program $P$ or program $Q$ on $G$.

The absence of cycles is checked by deleting, as long as possible, edges whose source nodes have no incoming edges, and testing subsequently whether any edges remain. This method relies on the following invariant of the rule schema delete: for every step $G \Rightarrow$ delete $H, G$ is acyclic if and only if $H$ is acyclic. Moreover, a graph to which delete is not applicable is acyclic if and only if it does not contain edges. Note that the condition of delete uses the new indegree function.

Example 3 (Recognising series-parallel graphs). Series-parallel graphs are inductively defined as follows. Every graph $G$ consisting of two nodes connected by an edge is series-parallel, where the edge's source and target are the source and target of $G$. Given series-parallel graphs $G$ and $H$, the graphs obtained from the disjoint union $G+H$ by the following two operations are also series-parallel. Serial composition: merge the target of $G$ with the source of $H$; the source of $G$ becomes the new source and the target of $H$ becomes the new target. Parallel composition: merge the source of $G$ with the source of $H$, and the target of $G$ with the target of $H$; sources and targets are preserved. 


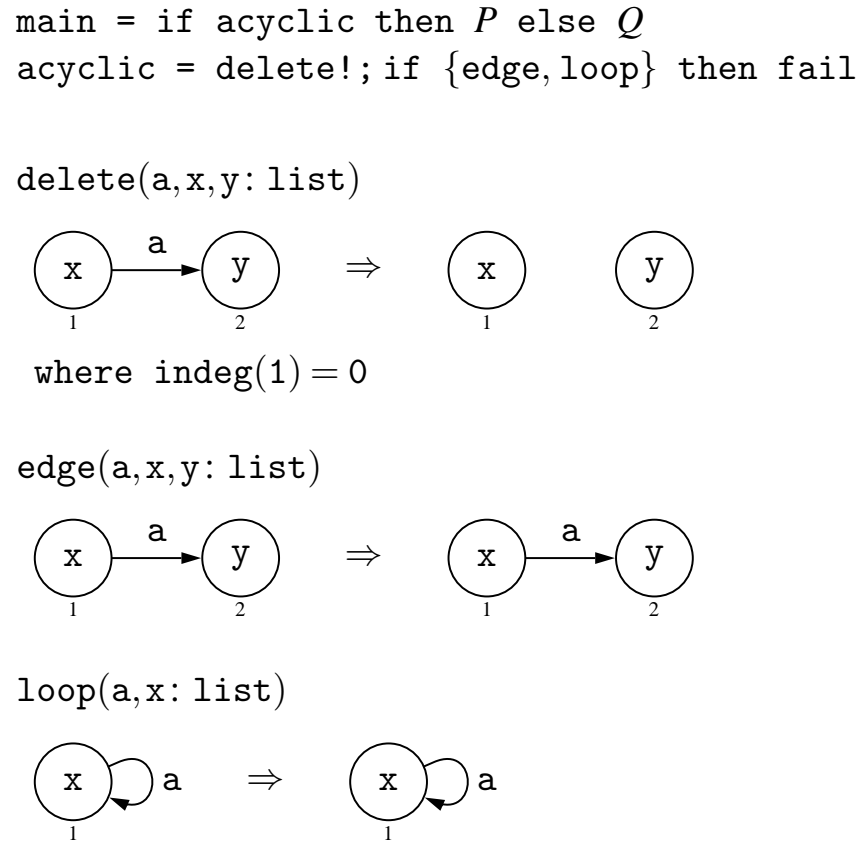

Figure 9: A program for recognising acyclic graphs

It is known [1, 2] that a graph is series-parallel if and only if it reduces to a graph consisting of two nodes connected by an edge (a base graph) by repeated application of the following operations: (a) Given a node with one incoming edge $i$ and one outgoing edge $o$ such that $s(i) \neq t(o)$, replace $i, o$ and the node by an edge from $s(i)$ to $t(o)$. (b) Replace a pair of parallel edges by an edge from their source to their target.

Figure 10 shows a macro which reduces every unmarked series-parallel graph to the empty graph, and fails on every other unmarked graph. The subprogram reduce! applies as long as possible the operations (a) and (b) to the input graph $G$, then the rule schema delete-base checks if the result is a base graph whose nodes are not incident to other edges. (The latter is ensured by the dangling condition.) If delete-base is not applicable, then the input graph was not reduced to a base graph. In this case the input graph is not series-parallel because every execution of reduce! yields the same graph. (This is because the critical pairs of the rule schemata serial and parallel are strongly joinable [6].)

Finally, after delete-base has been applied, the rule schema nonempty checks whether the graph resulting from reduce! contains nodes other than those of the base graph. The input graph is seriesparallel if and only if this is not the case.

Example 4 (Computing Euler cycles). An Euler cycle is a directed cycle of distinct edges that contains all edges and nodes of a graph. A graph is eulerian if it contains an Euler cycle. It is known that a graph is eulerian if and only if it is connected and each node has the same indegree as outdegree [1]. Based on this characterisation, the macro eulerian of Figure 11 checks whether an unmarked graph is eulerian or not. It does this by using the macro disconnected of Figure 8 and the new indegree and outdegree functions.

Given an unmarked eulerian input graph with atomic labels, the program in Figure 12 computes an Euler cycle and numbers its edges. An execution of this program is shown in Figure 13 In the resulting 


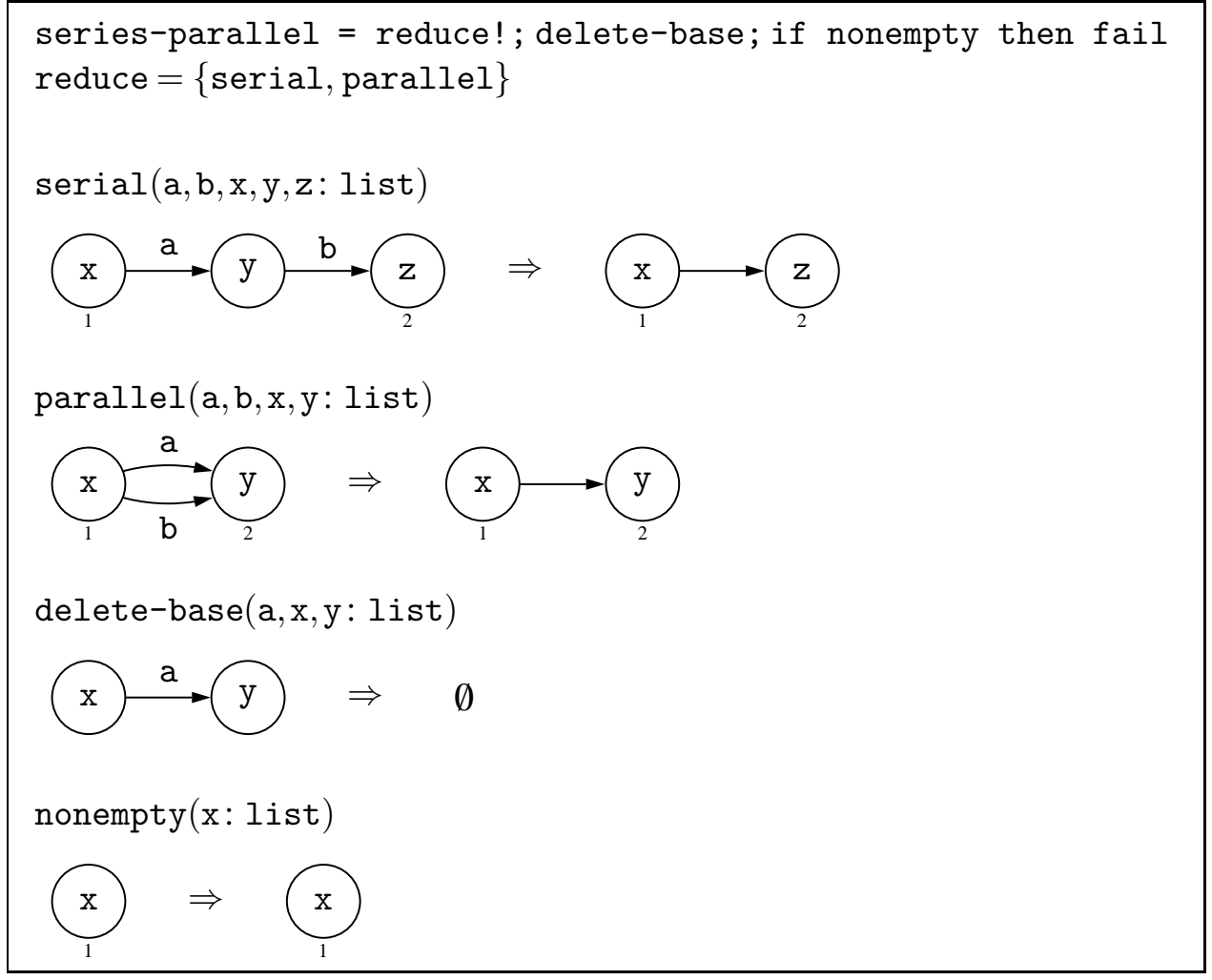

Figure 10: A macro for recognising series-parallel graphs

graph, the computed Euler cycle is given by the edges with the labels $1: 1,1: 1: 1,1: 1: 2,1: 1: 3,1: 2$, $1: 3$ and $1: 4$. The graph in the middle of Figure 13 is an intermediate result representing the point in time when the macro cycle has been executed for the first time.

The program uses the command try_then to check if the input graph is nonempty. If the input graph is empty, then the empty sequence of edges is an Euler cycle and hence the program returns the empty graph. If the input graph is nonempty, the rule schema init picks some node, adds 0 to its label, and marks the node. Then the rule schema loop numbers all loops with atomic labels that are incident to the node. Next the rule schema cycle numbers a proper (that is, non-loop) cycle starting at this node, by repeatedly applying the rule schema grow. Also, at each visited node, loop is applied as long as possible to number all incident loops.

When the first proper cycle has been numbered, the subprogram (next; cycle)! repeatedly computes a new cycle starting at a node that has already been visited. This cycle is inserted into the current cycle by numbering the new edges with lists that add one position to the list of the edge preceding the new edges. Finally, when all edges of the graph have been numbered, the rule schema clean-up removes all auxiliary information in node labels.

\section{Operational Semantics}

This section presents a formal semantics for GP 2 in the style of Plotkin's structural operational semantics [5]. As usual for this approach, inference rules inductively define a small-step transition relation $\rightarrow$ on 


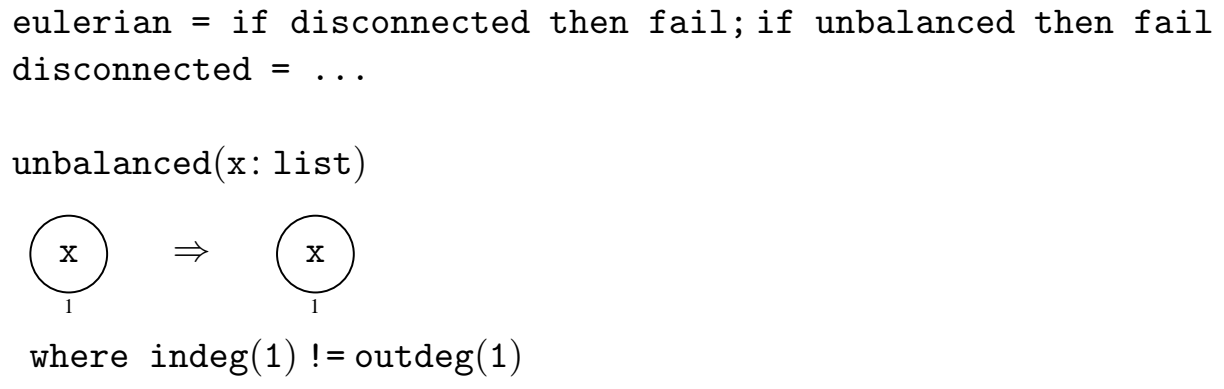

Figure 11: A macro for recognising eulerian graphs

configurations. In our setting, a configuration is either a command sequence together with a graph, just a graph or the special element fail:

$$
\rightarrow \subseteq(\mathrm{ComSeq} \times \mathscr{G}(\mathscr{L})) \times((\mathrm{ComSeq} \times \mathscr{G}(\mathscr{L})) \cup \mathscr{G}(\mathscr{L}) \cup\{\text { fail }\})
$$

Configurations in ComSeq $\times \mathscr{G}(\mathscr{L})$, given by a rest program and a state in the form of a graph, represent states of unfinished computations while graphs in $\mathscr{G}(\mathscr{L})$ are proper results. In addition, the element fail represents a failure state. A configuration $\gamma$ is said to be terminal if there is no configuration $\delta$ such that $\gamma \rightarrow \delta$.

Figure 14 in the Appendix shows the inference rules for the core commands of GP 2. Each rule consists of a premise and a conclusion separated by a horizontal bar. Both parts contain meta-variables for command sequences and graphs, where $R$ stands for a call in category RuleSetCall, $C, P, P^{\prime}, Q$ stand for command sequences in category ComSeq, and $G, H$ stand for graphs in $\mathscr{G}(\mathscr{L})$. Meta-variables are considered to be universally quantified. For example, the rule [call $\left.{ }_{1}\right]$ reads: "For all $R$ in RuleSetCall and all $G, H$ in $\mathscr{G}(\mathscr{L}), G \Rightarrow_{R} H$ implies $\langle R, G\rangle \rightarrow H$." The transitive and reflexive-transitive closures of $\rightarrow$ are written $\rightarrow^{+}$and $\rightarrow^{*}$, respectively. The notation $G \neq_{R}$ expresses that for graph $G$ in $\mathscr{G}(\mathscr{L})$ there is no graph $H$ such that $G \Rightarrow_{R} H$.

The if-then-else command has been designed to "hide" destructive tests. In Example1 for instance, the test of the if-then-else command produces a graph with marked nodes. By the inference rules $\left[\mathrm{if}_{1}\right]$ and [if 2 , this graph is discarded and program $P$ or $Q$ is executed on the input graph. In contrast, a program try $C$ then $P$ else $Q$ passes any graph resulting from its test to $P$. If test $C$ fails, however, $Q$ is executed on the input graph.

The semantics of the if-then-else command and the as-long-as-possible loop in GP 1 have been modified to allow an efficient implementation. Previously, the conditions of branching commands and the bodies of loops were tested, in the worst case, by trying all possible executions starting from the current graph. This made branching and loop commands impractical for complex tests or large input graphs. In GP 2, the semantics of if $C$ then $P$ else $Q$, try $C$ then $P$ else $Q$, and $B$ ! do not enforce backtracking when $C$ or $B$ fails. Instead, control is passed to program $Q$ or the loop is terminated, respectively. Note that this change increases the nondeterminism of evaluation in cases where $C$ or $B$ can both succeed and fail on the input graph.

The inference rules for the remaining GP commands are given in Figure 15 of the Appendix. These commands are referred to as derived commands because they can be defined by the core commands, as shown below.

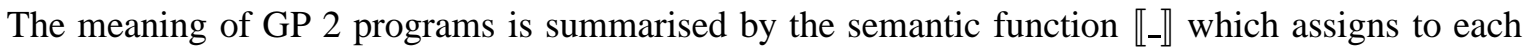
program $P$ the function $\llbracket P \rrbracket$ mapping an input graph $G$ to the set of all possible results of executing $P$ 


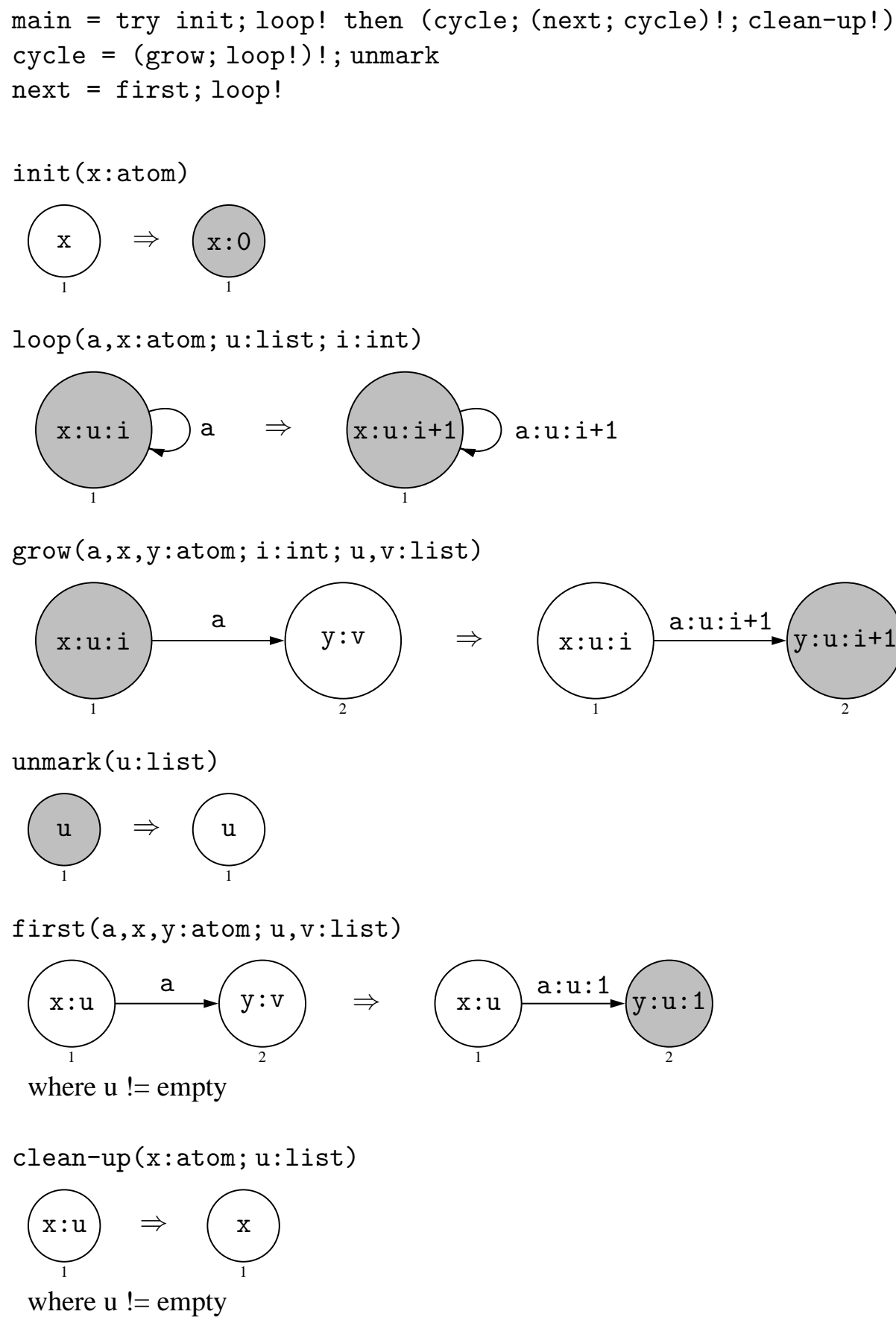

Figure 12: A program for computing an Euler cycle 


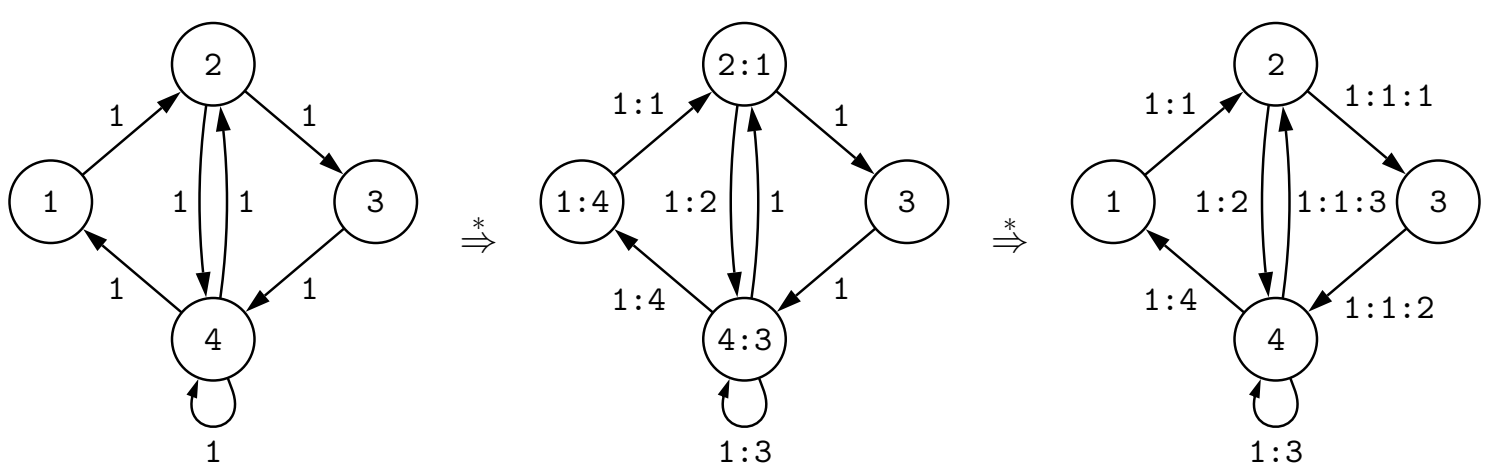

Figure 13: An execution of the program of Figure 12

on $G$. The application of $\llbracket P \rrbracket$ to $G$ is written $\llbracket P \rrbracket G$. The result set may contain, besides proper results in the form of graphs, the special values fail and $\perp$. The value fail indicates a failed program run while $\perp$ indicates a run that does not terminate or gets stuck. Program $P$ can diverge from $G$ if there is an infinite sequence $\langle P, G\rangle \rightarrow\left\langle P_{1}, G_{1}\right\rangle \rightarrow\left\langle P_{2}, G_{2}\right\rangle \rightarrow \ldots$ Also, $P$ can get stuck from $G$ if there is a terminal configuration $\langle Q, H\rangle$ such that $\langle P, G\rangle \rightarrow^{*}\langle Q, H\rangle$.

Definition 5 (Semantic function). The semantic function $\llbracket-\rrbracket:$ ComSeq $\rightarrow\left(\mathscr{G}(\mathscr{L}) \rightarrow 2^{\mathscr{G}(\mathscr{L}) \cup\{\text { fail, } \perp\}}\right)$ is defined by

$$
\llbracket P \rrbracket G=\{X \in(\mathscr{G}(\mathscr{L}) \cup\{\text { fail }\}) \mid\langle P, G\rangle \stackrel{+}{\rightarrow} X\} \cup\{\perp \mid P \text { can diverge or get stuck from } G\} .
$$

In the current implementation of GP, reaching the failure state triggers backtracking which then attempts to find a proper result [4]. However, backtracking can be switched off by the user.

A program can get stuck in two situations: (1) it contains a command if $C$ then $P$ else $Q$ or try $C$ then $P$ else $Q$ such that $C$ can diverge from some graph $G$ and can neither produce a proper result from $G$ nor fail from $G$, or (2) it contains a loop $B$ ! whose body $B$ possesses the said property of $C$. The evaluation of such commands gets stuck because none of the inference rules for if-then-else, try-then-else or iteration is applicable.

The semantic function of Definition 5 suggests a straightforward notion of program equivalence.

Definition 6 (Semantic equivalence). Two programs $P$ and $Q$ are semantically equivalent, denoted by $P \equiv Q$, if $\llbracket P \rrbracket=\llbracket Q \rrbracket$.

For example, it is easy to see that the following equivalences between derived commands and core commands hold (where $\emptyset$ is the empty graph):

- $\operatorname{skip} \equiv$ null, where null is the rule schema $\emptyset \Rightarrow \emptyset$;

- fail $\equiv\{\}$, where \{\} is the empty set of rule schemata;

- if $C$ then $P \equiv$ if $C$ then $P$ else null, for all programs $C$ and $P$;

- try $C$ then $P \equiv \operatorname{try} C$ then $P$ else null, for all programs $C$ and $P$.

Less obvious is the following equivalence, showing that or is a derived command:

$$
P \text { or } Q \equiv \text { if remove!; }\{\text { create, null\}; zero then } P \text { else } Q,
$$

for all programs $P$ and $Q$. Here remove is a set of three rule schemata that delete arbitrary edges, loops and isolated nodes, create is the rule schema

$$
\emptyset \Rightarrow \text { (0) }
$$


and zero is the rule schema

$$
\text { (0) } \Rightarrow \text { (0). }
$$

The following non-equivalence may be surprising, too:

$$
\operatorname{try} C \text { then } P \text { else } Q \not \equiv \text { if } C \text { then } C ; P \text { else } Q \text {. }
$$

To witness, choose $C=$ skip or fail, $P=$ skip and $Q=$ skip. Then the try-program is equivalent to skip and hence cannot fail, but the if-program can fail.

\section{Conclusion}

GP allows high-level problem solving in the domain of graphs, by supporting rule-based programming and freeing programmers from dealing with low-level data structures for graphs. The language has a simple syntax and semantics, to facilitate both understanding by programmers and formal reasoning on programs.

The revised language GP 2 has an improved type system, including list variables and subtypes, a new concept of marking nodes and edges graphically, new built-in functions for accessing the indegree and the outdegree of nodes, a more powerful edge predicate for conditions, new commands try-then-else and or, and a simplified semantics of branching and looping to enable an efficient implementation.

Topics for future work include the implementation of GP 2, tool support for Hoare-style program verification [9], and static analyses for properties such as termination and confluence.

Acknowledgements. Parts of this paper were written while visiting Annegret Habel in Oldenburg and Berthold Hoffmann in Bremen in the autumn of 2011. I am grateful for their hospitality. Thanks go also to the Plasma research group in York for helpful comments, especially to Colin Runciman for proposing the concept of shaded nodes.

\section{References}

[1] Jørgen Bang-Jensen \& Gregory Gutin (2009): Digraphs: Theory, Algorithms and Applications, second edition. Springer-Verlag.

[2] R. J. Duffin (1965): Topology of Series-Parallel Networks. Journal of Mathematical Analysis and Applications 10(2), pp. 303-318, doi:10.1016/0022-247X(65)90125-3

[3] Annegret Habel \& Detlef Plump (2002): Relabelling in Graph Transformation. In: Proc. International Conference on Graph Transformation (ICGT 2002), Lecture Notes in Computer Science 2505, Springer-Verlag, pp. 135-147, doi:10.1007/3-540-45832-8_12

[4] Greg Manning \& Detlef Plump (2008): The GP Programming System. In: Proc. Graph Transformation and Visual Modelling Techniques (GT-VMT 2008), Electronic Communications of the EASST 10.

[5] Gordon D. Plotkin (2004): A Structural Approach to Operational Semantics. Journal of Logic and Algebraic Programming 60-61, pp. 17-139, doi:10.1016/j.jlap.2004.05.001

[6] Detlef Plump (2005): Confluence of Graph Transformation Revisited. In Aart Middeldorp, Vincent van Oostrom, Femke van Raamsdonk \& Roel de Vrijer, editors: Processes, Terms and Cycles: Steps on the Road to Infinity: Essays Dedicated to Jan Willem Klop on the Occasion of His 60th Birthday, Lecture Notes in Computer Science 3838, Springer-Verlag, pp. 280-308, doi:10.1007/11601548 
[7] Detlef Plump (2009): The Graph Programming Language GP. In: Proc. International Conference on Algebraic Informatics (CAI 2009), Lecture Notes in Computer Science 5725, Springer-Verlag, pp. 99-122, doi:10.1007/978-3-642-03564-7_6.

[8] Detlef Plump \& Sandra Steinert (2010): The Semantics of Graph Programs. In: Proc. Rule-Based Programming (RULE 2009), Electronic Proceedings in Theoretical Computer Science 21, pp. 27-38, doi $10.4204 /$ EPTCS.21.3

[9] Christopher M. Poskitt \& Detlef Plump (2012): Hoare-Style Verification of Graph Programs. Fundamenta Informaticae. To appear.

\section{Appendix: Semantic Inference Rules}

$$
\begin{aligned}
& {\left[\text { call }_{1}\right] \frac{G \Rightarrow_{R} H}{\langle R, G\rangle \rightarrow H}} \\
& {\left[\mathrm{call}_{2}\right] \frac{G \neq_{R}}{\langle R, G\rangle \rightarrow \text { fail }}} \\
& {\left[\text { seq }_{1}\right] \frac{\langle P, G\rangle \rightarrow\left\langle P^{\prime}, H\right\rangle}{\langle P ; Q, G\rangle \rightarrow\left\langle P^{\prime} ; Q, H\right\rangle}} \\
& {\left[\operatorname{seq}_{2}\right] \frac{\langle P, G\rangle \rightarrow H}{\langle P ; Q, G\rangle \rightarrow\langle Q, H\rangle}} \\
& {\left[\mathrm{seq}_{3}\right] \frac{\langle P, G\rangle \rightarrow \text { fail }}{\langle P ; Q, G\rangle \rightarrow \text { fail }}} \\
& {\left[\text { if }_{1}\right] \frac{\langle C, G\rangle \rightarrow^{+} H}{\langle\text { if } C \text { then } P \text { else } Q, G\rangle \rightarrow\langle P, G\rangle}} \\
& {\left[\text { if }_{2}\right] \frac{\langle C, G\rangle \rightarrow^{+} \text {fail }}{\langle\text { if } C \text { then } P \text { else } Q, G\rangle \rightarrow\langle Q, G\rangle}} \\
& {\left[\operatorname{try}_{1}\right] \frac{\langle C, G\rangle \rightarrow^{+} H}{\langle\operatorname{try} C \text { then } P \text { else } Q, G\rangle \rightarrow\langle P, H\rangle}} \\
& {\left[\operatorname{try}_{2}\right] \frac{\langle C, G\rangle \rightarrow^{+} \text {fail }}{\langle\operatorname{try} C \text { then } P \text { else } Q, G\rangle \rightarrow\langle Q, G\rangle}} \\
& {\left[\operatorname{alap}_{1}\right] \frac{\langle P, G\rangle \rightarrow^{+} H}{\langle P !, G\rangle \rightarrow\langle P !, H\rangle}} \\
& {\left[\operatorname{alap}_{2}\right] \frac{\langle P, G\rangle \rightarrow^{+} \text {fail }}{\langle P !, G\rangle \rightarrow G}}
\end{aligned}
$$

Figure 14: Inference rules for core commands

$$
\begin{array}{ll}
{\left[\text { or }_{1}\right]\langle P \text { or } Q, G\rangle \rightarrow\langle P, G\rangle} & {\left[\text { or }_{2}\right]\langle P \text { or } Q, G\rangle \rightarrow\langle Q, G\rangle} \\
\text { [skip] }\langle\text { skip }, G\rangle \rightarrow G & {[\text { fail] }\langle\text { fail, } G\rangle \rightarrow \text { fail }} \\
{\left[\text { if }_{3}\right] \frac{\langle C, G\rangle \rightarrow^{+} H}{\langle\text { if } C \text { then } P, G\rangle \rightarrow\langle P, G\rangle}} & {\left[\text { if }_{4}\right] \frac{\langle C, G\rangle \rightarrow^{+} \text {fail }}{\langle\text { if } C \text { then } P, G\rangle \rightarrow G}} \\
{\left[\operatorname{try}_{3}\right] \frac{\langle C, G\rangle \rightarrow^{+} H}{\langle\operatorname{try} C \operatorname{then} P, G\rangle \rightarrow\langle P, H\rangle}} & {\left[\operatorname{try}_{4}\right] \frac{\langle C, G\rangle \rightarrow^{+} \text {fail }}{\langle\operatorname{try} C \operatorname{then} P, G \rightarrow G}}
\end{array}
$$

Figure 15: Inference rules for derived commands 